\title{
Toxic Elements in Commercial Infant Food, Estimated Dietary Intake, and Risk Assessment in Poland
}

\author{
Monika Mania*, Maria Wojciechowska-Mazurek, Krystyna Starska, \\ Malgorzata Rebeniak, Tomasz Szynal, Agnieszka Strzelecka, \\ Jacek Postupolski
}

National Institute of Public Health - National Institute of Hygiene (NIPH - NIH), Chocimska 24, 00-791 Warsaw, Poland

Received: July 31, 2014

Accepted: September 25, 2015

\begin{abstract}
This paper presents results of heavy metals determination in infant foods available in Poland during a monitoring programme in 2009-13. This study investigated levels of lead, cadmium, arsenic, and mercury in infant formulae and other infant foods intended for consumption during the first 6 to 12 months of life, and an intake of heavy metals was assessed. Food consumption data based on the artificial feeding scheme of infants established by the Mother and Child Institute in Poland were taken into account to estimate dietary exposure. The reported levels of elements in infant foods are comparable with contamination of such products in other countries. The mean values for lead do not exceed $0.013 \mathrm{mg} / \mathrm{kg}\left(90^{\text {th }}\right.$ percentile, $\left.0.028 \mathrm{mg} / \mathrm{kg}\right)$. Lead levels in infant formulae (as sold, mainly powder) did not exceed $0.010 \mathrm{mg} / \mathrm{kg}$, while mean contamination was $0.005 \mathrm{mg} / \mathrm{kg}$. The highest mean cadmium level of $0.010 \mathrm{mg} / \mathrm{kg}$ was reported in vegetable meal $\left(90^{\text {th }}\right.$ percentile, $0.017 \mathrm{mg} / \mathrm{kg}$ ); in infant formulae (undiluted) milk-based it was below $0.003 \mathrm{mg} / \mathrm{kg}$, and for soya-based it was $0.009 \mathrm{mg} / \mathrm{kg}$. Arsenic and mercury contents - highest in fish-based infant foods - did not exceed $0.18 \mathrm{mg} / \mathrm{kg}$ and $0.013 \mathrm{mg} / \mathrm{kg}$, respectively. In rice products for infants arsenic did not exceed $0.14 \mathrm{mg} / \mathrm{kg}$. The estimated mean lead exposure was from $0.09 \mu \mathrm{g} / \mathrm{kg}$ b.w. per day for 6-month-old infants to $0.53 \mu \mathrm{g} / \mathrm{kg}$ b.w. per day for 1 -year-old children, which was approximately $17 \%$ and equal to the respective Benchmark Dose Lower Confidence Limit $\left(\mathrm{BMDL}_{01}\right)$ established by the European Food Safety Authority (EFSA) at $0.50 \mu \mathrm{g} / \mathrm{kg}$ b.w. per day. Mean exposure to cadmium ranges from 0.02 to $0.4 \mu \mathrm{g} / \mathrm{kg}$ b.w. per day, and for 12-month-old infants can slightly exceed the tolerable weekly intake (TWI) value established by EFSA. Dietary cadmium intake represents $2.5-47 \%$ of the provisional tolerable monthly intake (PTMI) value established by the Joint FAO/WHO Expert Committee of Food Additives (JECFA). Intake of arsenic and mercury was below respective reference value. Calculated mean dietary exposure to arsenic for children under 1 year old was between 0.11 and $0.99 \mu \mathrm{g} / \mathrm{kg}$ b.w. per day (4-33\% $\mathrm{BMDL}_{0.5}$ value), and to methylmercury $0.01-0.08 \mu \mathrm{g} / \mathrm{kg}$ b.w. per day (7-41\% of the TWI value for methylmercury).
\end{abstract}

Keywords: heavy metals, risk assessment, infant foods, dietary intake

*e-mail: mmania@pzh.gov.pl 


\section{Introduction}

Infants are the population group most vulnerable to the toxic effects of heavy metals due to the higher absorption of metals by the gastrointestinal tract, faster metabolic processes, an incompletely developed detoxification system, and higher food consumption in relation to body weight [1-3]. Additionally, the undeveloped blood-brain barrier allows elements noxious to infant health (primarily lead and mercury compounds) to accumulate in the brain, causing dysfunction of the central nervous system [4]. Exposure to heavy metals during growth and development can result in long-term effects on the health of children [5]. Infant foods are the main source of heavy metals intake by this population, primarily due to contamination of raw materials used and rarely by food processing itself [6-8].

Commercially available infant foods have become an important part of the diet of many infants and toddlers because of their mineral and vitamin content that fulfills dietary requirements of these target groups [9-11]. The European Commission has defined specific maximum levels for toxic elements in foodstuffs designated for this population group, only for lead in infant formulae and ready-to-use follow-on formulae at $0.02 \mathrm{mg} / \mathrm{kg}$, cadmium in processed cereal-based foods, baby foods for infants and young children at $0.04 \mathrm{mg} / \mathrm{kg}$, and in infant formulae and follow-on formulae in the range $0.005-0.02 \mathrm{mg} / \mathrm{kg}$, depending of the type of products (Commission Regulation 1881/2006 of 19 December 2006 setting maximum levels for certain contaminants in foodstuffs, as amended; last amendment 12 May 2014).

In 2010 the Panel on Contaminants in the Food Chain (CONTAM) of the European Food Safety Authority (EFSA) identified lead for developmental neurotoxicity and cardiovascular effects in young children, and nephrotoxicity in adults as the critical effects for the risk assessment. The respective benchmark dose lower confidence limits (BMDLs) established by EFSA were:

- For developmental neurotoxicity in young children $\mathrm{BMDL}_{01}, 0.50 \mu \mathrm{g} / \mathrm{kg}$ body weight (b.w.) per day

- Effects on systolic blood pressure in adults $\mathrm{BMDL}_{01}$, $1.50 \mu \mathrm{g} / \mathrm{kg}$ b.w. per day

- Effects on prevalence of chronic kidney disease in adults $\mathrm{BMDL}_{10}, 0.63 \mu \mathrm{g} / \mathrm{kg}$ b.w. per day

The CONTAM Panel concluded that the current provisional tolerable weekly intake (PTWI) at $25 \mu \mathrm{g} / \mathrm{kg}$ b.w. is no longer appropriate as there is no evidence for the existence of a threshold for critical lead-induced effects and withdrew that value. In children, an elevated blood lead level is inversely associated with a reduced intelligence quotient (IQ) [12]. The Joint FAO/WHO Expert Committee on Food Additives (JECFA) also withdrew the PTWI value based on dose-response analyses and did not establish a new level, which could be considered a health protective [13].

Moreover, EFSA established a tolerable weekly intake (TWI) for cadmium at $2.5 \mu \mathrm{g} / \mathrm{kg}$ b.w. [14]. In 2010, JECFA considering the long half-life of cadmium, and taking into account the negligible effect of daily exposure on overall exposure, decided to express the tolerable intake as a monthly value of provisional tolerable monthly intake (PTMI) at $25 \mu \mathrm{g} / \mathrm{kg}$ b.w., which was not in line with the EFSA risk assessment [13].

For arsenic, the CONTAM panel of EFSA established $\mathrm{BMDL}_{01}$ values between 0.3 and $8 \mu \mathrm{g} / \mathrm{kg}$ b.w. per day for lung, skin, and bladder cancer, as well as skin lesions [15]. JECFA for arsenic computed BMDL $\mathrm{BM}_{0.5}$ to be $3.0 \mu \mathrm{g} / \mathrm{kg}$ b.w. per day $(2.0 \mu \mathrm{g} / \mathrm{kg}$ b.w. per day $-7.0 \mu \mathrm{g} / \mathrm{kg}$ b.w. per day) [16].

In 2010, JECFA adopted PTWI for inorganic mercury at $4.0 \mu \mathrm{g} / \mathrm{kg}$ and for organic mercury at $1.6 \mu \mathrm{g} / \mathrm{kg} \mathrm{b}$.w. [16]. In the final opinion of 2012, EFSA established a TWI for methylmercury at $1.3 \mu \mathrm{g} / \mathrm{kg}$ b.w. [17].

This paper presents results of detailed investigations performed for infant foods intended for consumption during the first six and 12 months of life. Obtained data were used to perform specific exposure assessments associated with intake of toxic elements by one of the most vulnerable target groups of the population.

\section{Experimental Procedures}

\section{Samples}

Approximately 1,000 samples of commercially available products for infants were sampled and tested for lead, cadmium, mercury, and arsenic levels by sanitary-epidemiological stations during the monitoring program, prepared and coordinated by the Department of Food Safety in the National Institute of Public Health-National Institute of Hygiene (NIPH-NIH) and performed in Poland in 2009-13. Samples were taken in all provinces in Poland from the retail market in line with NIPH-NIH guidelines and in accordance with requirements of Commission Regulation No. 333/2007 of 28 March 2007 laying down the methods of sampling and analysis for official control of the levels of lead, cadmium, mercury, inorganic tin, 3-MCPD, and benzo(a)pyrene in foodstuffs, amended by Regulation No. 836/2011.

The products under analysis were: infant formulae and follow-on formulae samples, soya-based infant formulae; milk- and rice-based products for infants; rice gruel, wheat gruel, and mixed cereals for infants (all these products were sold mainly as powder); ready-to-eat puréed infant foods including vegetable meals, vegetable and meat meals, fishbased infant food, and fruit-based desserts; and juices, biscuits, herbal teas, and teas with herbs and fruits. Samples from domestic production dominated, along with products from other EU Member States (i.e., France, Germany, the Netherlands, Czech Republic, Belgium, Spain) which comprised about $30 \%$ of the total. All samples were analysed as sold (they were not reconstituted prior to analysis).

\section{Sample Preparation}

Samples were treated by using different methods of digestion depending on the element investigated. For lead and cadmium determination, samples were prepared by microwave digestion according to: EN 14083:2003 or by dry ashing based on EN 14082:2003, and for arsenic deter- 
mination by dry ashing according to EN 14546:2005. Samples for mercury determination were analyzed directly or after pressure digestion according to EN 13806:2002.

\section{Trace Elements Analysis}

Contents of elements noxious to health were determined using atomic absorption spectrometry (AAS). Lead and cadmium were determined using flame atomic absorption spectrometry (FAAS) after extraction of metal complexes with ammonium pyrrolidine dithiocarbamate (APDC) to organic solvent according to Methodology Publications of the National Institute of Hygiene [18] or graphite furnace atomic absorption spectrometry (GFAAS). Arsenic was determined using hydride generation atomic absorption spectrometry (HGAAS), and mercury was determined using the "cold vapour" technique (CVAAS).

\section{Quality Assurance}

Validated analytical methods meeting criteria set in the legislation were applied. Laboratories of SanitaryEpidemiological Stations participating in these studies are accredited according to EN ISO/IEC 17025:2005 and apply internal and external quality procedures. Quality control involved the use of reference materials with certified contents of metals. The laboratories also verified their proficiency in these methods by participating in interlabolatory proficiency tests organized by, i.e., the Laboratory of the Department of Food Safety - National Reference Laboratory located in NIPH-NIH (Poland) and the Food and Environment Research Agency (UK) - Food Analysis Performance Assessment Scheme (FAPAS). The NIPHNIH Reference Laboratory itself participates in proficiency tests organized by, i.e., the European Union Reference Laboratory Heavy Metals in Feed and Food (EU-RL-HM) - Joint Research Centre, Institute for Reference Materials and Measurements, Belgium (JRC-IRMM) and FAPAS.

\section{Statistical Evaluation}

Statistical assessment of results was performed according the substitution method used by EFSA for the treatment of left-censored data - LC (below limit of detection (LOD)). For results reported to be below the LOD, the value equal to the LOD (upper bound - UB), zero (lower bound $-\mathrm{LB}$ ) or half the LOD (medium or middle bound MB) were used [19]. Different numbers of results below LOD were observed in the analyzed samples. Depending on element and foodstuff, left-censored data (LC) were between $90 \%$ for mercury in herbal and fruit teas and 5\% for cadmium in vegetable meals.

\section{Results and Discussion}

Concentrations of the investigated elements in the analyzed samples of infant foods as sold are presented in Tables 1-4. The concentrations of toxic elements are presented as a mean, median, and $90^{\text {th }}$ percentile (P90), lower (LB), middle (MB) and upper bound (UB).
Table 1. Lead content in different types of commercially available infant foods in the Polish market ( $\mathrm{mg} / \mathrm{kg}$ ).

\begin{tabular}{|c|c|c|c|c|}
\hline \multirow{2}{*}{ Product } & \multicolumn{4}{|c|}{$\mathrm{Pb}$} \\
\hline & $\mathrm{n}$ & Mean & Median & P90 \\
\hline \multirow{3}{*}{$\begin{array}{l}\text { Infant } \\
\text { formula, } \\
\text { follow-on } \\
\text { formula }\end{array}$} & \multirow{3}{*}{112} & LB: 0.0024 & LB: 0.0000 & LB: 0.0065 \\
\hline & & MB: 0.0051 & MB: 0.0050 & MB: 0.0099 \\
\hline & & UB: 0.0077 & UB: 0.0080 & UB: 0.0118 \\
\hline \multirow{3}{*}{$\begin{array}{l}\text { Milk and } \\
\text { rice-based } \\
\text { products }\end{array}$} & \multirow{3}{*}{58} & LB: 0.0024 & LB: 0.0000 & LB: 0.0066 \\
\hline & & MB: 0.0046 & MB: 0.0048 & MB: 0.0100 \\
\hline & & UB: 0.0069 & UB: 0.0050 & UB: 0.0200 \\
\hline \multirow{3}{*}{$\begin{array}{c}\text { Infant formula } \\
\text { soya-based }\end{array}$} & \multirow{3}{*}{22} & LB: 0.0037 & LB: 0.0000 & LB: 0.0089 \\
\hline & & MB: 0.0060 & MB: 0.0053 & MB: 0.0099 \\
\hline & & UB: 0.0082 & UB: 0.0070 & UB: 0.0155 \\
\hline \multirow{3}{*}{$\begin{array}{l}\text { Rice gruel } \\
\text { for infants }\end{array}$} & \multirow{3}{*}{185} & LB: 0.0054 & LB: 0.0000 & LB: 0.0146 \\
\hline & & MB: 0.0083 & MB: 0.0050 & MB: 0.0146 \\
\hline & & UB: 0.0112 & UB: 0.0100 & UB: 0.0200 \\
\hline \multirow{3}{*}{$\begin{array}{l}\text { Wheat gruel } \\
\text { for infants }\end{array}$} & \multirow{3}{*}{74} & LB: 0.0030 & LB: 0.0000 & LB: 0.0100 \\
\hline & & MB: 0.0056 & MB: 0.0048 & MB: 0.0100 \\
\hline & & UB: 0.0082 & UB: 0.0075 & UB: 0.0200 \\
\hline \multirow{3}{*}{$\begin{array}{l}\text { Mixed cereals } \\
\text { for infants }\end{array}$} & \multirow{3}{*}{65} & LB: 0.0040 & LB: 0.0000 & LB: 0.0090 \\
\hline & & MB: 0.0064 & MB: 0.0050 & MB: 0.0100 \\
\hline & & UB: 0.0089 & UB: 0.0090 & UB: 0.0192 \\
\hline \multirow{3}{*}{$\begin{array}{l}\text { Biscuits } \\
\text { for infants }\end{array}$} & \multirow{3}{*}{49} & LB: 0.0104 & LB: 0.0020 & LB: 0.0282 \\
\hline & & MB: 0.0126 & MB: 0.0050 & MB: 0.0282 \\
\hline & & UB: 0.0147 & UB: 0.0100 & UB: 0.0282 \\
\hline \multirow{3}{*}{$\begin{array}{l}\text { Vegetable } \\
\text { meals } \\
\text { ready-to-eat }\end{array}$} & \multirow{3}{*}{49} & LB: 0.0049 & LB: 0.0000 & LB: 0.0142 \\
\hline & & MB: 0.0072 & MB: 0.0050 & MB: 0.0142 \\
\hline & & UB: 0.0094 & UB: 0.0100 & UB: 0.0142 \\
\hline \multirow{3}{*}{$\begin{array}{l}\text { Vegetable- } \\
\text { meat meals } \\
\text { ready-to-eat }\end{array}$} & \multirow{3}{*}{64} & LB: 0.0054 & LB: 0.0000 & LB: 0.0148 \\
\hline & & MB: 0.0077 & MB: 0.0050 & MB: 0.0148 \\
\hline & & UB: 0.0100 & UB: 0.0100 & UB: 0.0185 \\
\hline \multirow{3}{*}{$\begin{array}{l}\text { Fish-based } \\
\text { infant foods } \\
\text { ready-to-eat }\end{array}$} & \multirow{3}{*}{47} & LB: 0.0053 & LB: 0.0040 & LB: 0.0110 \\
\hline & & MB: 0.0073 & MB: 0.0050 & MB: 0.0110 \\
\hline & & UB: 0.0093 & UB: 0.0100 & UB: 0.0110 \\
\hline \multirow{3}{*}{$\begin{array}{l}\text { Fruits desserts } \\
\text { for infants }\end{array}$} & \multirow{3}{*}{43} & LB: 0.0052 & LB: 0.0000 & LB: 0.0163 \\
\hline & & MB: 0.0075 & MB: 0.0050 & MB: 0.0163 \\
\hline & & UB: 0.0098 & UB: 0.0100 & UB: 0.0163 \\
\hline \multirow{3}{*}{$\begin{array}{l}\text { Juices } \\
\text { for infants }\end{array}$} & & LB: 0.0049 & LB: 0.0030 & LB: 0.0150 \\
\hline & 69 & MB: 0.0061 & MB: 0.0050 & MB: 0.0150 \\
\hline & & UB: 0.0074 & UB: 0.0050 & UB: 0.0150 \\
\hline & & LB: 0.0041 & LB: 0.0000 & LB: 0.0106 \\
\hline Herbal teas & 45 & MB: 0.0064 & MB: 0.0050 & MB: 0.0134 \\
\hline & & UB: 0.0086 & UB: 0.0100 & UB: 0.0150 \\
\hline Herbal and & & LB: 0.0046 & LB: 0.0007 & LB: 0.0106 \\
\hline fruit teas & 60 & MB: 0.0071 & MB: 0.0050 & MB: 0.0151 \\
\hline & & UB: 0.0096 & UB: 0.0080 & UB: 0.0237 \\
\hline
\end{tabular}

$\mathrm{n}$ - number of samples, LB - lower bound, MB - middle bound, UB - upper bound, $\mathrm{P} 90-90^{\text {th }}$ percentile 
Table 2. Cadmium content in different types of commercially available infant foods in the Polish market $(\mathrm{mg} / \mathrm{kg})$.

\begin{tabular}{|c|c|c|c|c|}
\hline \multirow{2}{*}{ Product } & \multicolumn{4}{|c|}{$\mathrm{Cd}$} \\
\hline & $\mathrm{n}$ & Mean & Median & P90 \\
\hline \multirow{3}{*}{$\begin{array}{l}\text { Infant } \\
\text { formula, } \\
\text { follow-on } \\
\text { formula }\end{array}$} & \multirow{3}{*}{119} & LB: 0.0010 & LB: 0.0000 & LB: 0.0030 \\
\hline & & MB: 0.0012 & MB: 0.0006 & MB: 0.0030 \\
\hline & & UB: 0.0015 & UB: 0.0010 & UB: 0.0030 \\
\hline \multirow{3}{*}{$\begin{array}{l}\text { Milk and } \\
\text { rice-based } \\
\text { products }\end{array}$} & \multirow{3}{*}{62} & LB: 0.0014 & LB: 0.0010 & LB: 0.0032 \\
\hline & & MB: 0.0016 & MB: 0.0010 & MB: 0.0032 \\
\hline & & UB: 0.0017 & UB: 0.0014 & UB: 0.0032 \\
\hline \multirow{3}{*}{$\begin{array}{c}\text { Infant formula } \\
\text { soya-based }\end{array}$} & \multirow{3}{*}{22} & LB: 0.0033 & LB: 0.0020 & LB: 0.0089 \\
\hline & & MB: 0.0034 & MB: 0.0020 & MB: 0.0089 \\
\hline & & UB: 0.0036 & UB: 0.0020 & UB: 0.0089 \\
\hline \multirow{3}{*}{$\begin{array}{l}\text { Rice gruel } \\
\text { for infants }\end{array}$} & \multirow{3}{*}{198} & LB: 0.0038 & LB: 0.0017 & LB: 0.0113 \\
\hline & & MB: 0.0041 & MB: 0.0019 & MB: 0.0119 \\
\hline & & UB: 0.0044 & UB: 0.0020 & UB: 0.0119 \\
\hline \multirow{3}{*}{$\begin{array}{l}\text { Wheat gruel } \\
\text { for infants }\end{array}$} & \multirow{3}{*}{86} & LB: 0.0047 & LB: 0.0042 & LB: 0.0090 \\
\hline & & MB: 0.0047 & MB: 0.0042 & MB: 0.0090 \\
\hline & & UB: 0.0047 & UB: 0.0042 & UB: 0.0090 \\
\hline \multirow{3}{*}{$\begin{array}{l}\text { Mixed cereals } \\
\text { for infants }\end{array}$} & \multirow{3}{*}{74} & LB: 0.0034 & LB: 0.0030 & LB: 0.0077 \\
\hline & & MB: 0.0035 & MB: 0.0030 & MB: 0.0077 \\
\hline & & UB: 0.0036 & UB: 0.0030 & UB: 0.0077 \\
\hline \multirow{3}{*}{$\begin{array}{l}\text { Biscuits } \\
\text { for infants }\end{array}$} & \multirow{3}{*}{49} & LB: 0.0066 & LB: 0.0064 & LB: 0.0109 \\
\hline & & MB: 0.0066 & MB: 0.0064 & MB: 0.0109 \\
\hline & & UB: 0.0067 & UB: 0.0064 & UB: 0.0109 \\
\hline \multirow{3}{*}{$\begin{array}{l}\text { Vegetable } \\
\text { meals } \\
\text { ready-to-eat }\end{array}$} & \multirow{3}{*}{49} & LB: 0.0096 & LB: 0.0080 & LB: 0.0174 \\
\hline & & MB: 0.0096 & MB: 0.0080 & MB: 0.0174 \\
\hline & & UB: 0.0097 & UB: 0.0080 & UB: 0.0174 \\
\hline \multirow{3}{*}{$\begin{array}{l}\text { Vegetable- } \\
\text { meat meals } \\
\text { ready-to-eat }\end{array}$} & \multirow{3}{*}{64} & LB: 0.0069 & LB: 0.0060 & LB: 0.0133 \\
\hline & & MB: 0.0070 & MB: 0.0060 & MB: 0.0133 \\
\hline & & UB: 0.0070 & UB: 0.0060 & UB: 0.0133 \\
\hline \multirow{3}{*}{$\begin{array}{l}\text { Fish-based } \\
\text { infant foods } \\
\text { ready-to-eat }\end{array}$} & \multirow{3}{*}{47} & LB: 0.0066 & LB: 0.0061 & LB: 0.0119 \\
\hline & & MB: 0.0067 & MB: 0.0061 & MB: 0.0119 \\
\hline & & UB: 0.0067 & UB: 0.0061 & UB: 0.0119 \\
\hline \multirow{3}{*}{$\begin{array}{l}\text { Fruits desserts } \\
\text { for infants }\end{array}$} & \multirow{3}{*}{43} & LB: 0.0016 & LB: 0.0003 & LB: 0.0035 \\
\hline & & MB: 0.0018 & MB: 0.0006 & MB: 0.0035 \\
\hline & & UB: 0.0020 & UB: 0.0010 & UB: 0.0035 \\
\hline \multirow{3}{*}{$\begin{array}{l}\text { Juices } \\
\text { for infants }\end{array}$} & & LB: 0.0009 & LB: 0.0008 & LB: 0.0020 \\
\hline & 69 & MB: 0.0010 & MB: 0.0009 & MB: 0.0020 \\
\hline & & UB: 0.0012 & UB: 0.0010 & UB: 0.0020 \\
\hline & & LB: 0.0003 & LB: 0.0000 & LB: 0.0010 \\
\hline Herbal teas & 45 & MB: 0.0006 & MB: 0.0005 & MB: 0.0010 \\
\hline & & UB: 0.0009 & UB: 0.0008 & UB: 0.0016 \\
\hline Herbal and & & LB: 0.0009 & LB: 0.0000 & LB: 0.0027 \\
\hline & 60 & MB: 0.0011 & MB: 0.0005 & MB: 0.0027 \\
\hline & & UB: 0.0014 & UB: 0.0010 & UB: 0.0027 \\
\hline
\end{tabular}

$\mathrm{n}$ - number of samples, LB - lower bound, MB - middle bound, UB - upper bound, $\mathrm{P} 90-90^{\text {th }}$ percentile
Table 3. Arsenic content in different types of commercially available infant foods in the Polish market $(\mathrm{mg} / \mathrm{kg})$.

\begin{tabular}{|c|c|c|c|c|}
\hline \multirow{2}{*}{ Product } & \multicolumn{4}{|c|}{ As } \\
\hline & $\mathrm{n}$ & Mean & Median & P90 \\
\hline \multirow{3}{*}{$\begin{array}{l}\text { Infant } \\
\text { formula, } \\
\text { follow-on } \\
\text { formula }\end{array}$} & \multirow{3}{*}{118} & LB: 0.0029 & LB: 0.0000 & LB: 0.0073 \\
\hline & & MB: 0.0067 & MB: 0.0040 & MB: 0.0129 \\
\hline & & UB: 0,0105 & UB: 0.0050 & UB: 0.0240 \\
\hline \multirow{3}{*}{$\begin{array}{l}\text { Milk and } \\
\text { rice-based } \\
\text { products }\end{array}$} & \multirow{3}{*}{62} & LB: 0.0092 & LB: 0.0029 & LB: 0.0260 \\
\hline & & MB: 0.0121 & MB: 0.0100 & MB: 0.0260 \\
\hline & & UB: 0.0149 & UB: 0.0120 & UB: 0.0359 \\
\hline \multirow{3}{*}{$\begin{array}{c}\text { Infant formula } \\
\text { soya-based }\end{array}$} & \multirow{3}{*}{22} & LB: 0.0096 & LB: 0.0065 & LB: 0.0210 \\
\hline & & MB: 0.0110 & MB: 0.0070 & MB: 0.0210 \\
\hline & & UB: 0.0123 & UB: 0.0100 & UB: 0.0210 \\
\hline \multirow{3}{*}{$\begin{array}{l}\text { Rice gruel } \\
\text { for infants }\end{array}$} & \multirow{3}{*}{197} & LB: 0.0585 & LB: 0.0450 & LB: 0.1404 \\
\hline & & MB: 0.0595 & MB: 0.0450 & MB: 0.1404 \\
\hline & & UB: 0.0605 & UB: 0.0450 & UB: 0.1404 \\
\hline \multirow{3}{*}{$\begin{array}{l}\text { Wheat gruel } \\
\text { for infants }\end{array}$} & \multirow{3}{*}{86} & LB: 0.0034 & LB: 0.0000 & LB: 0.0120 \\
\hline & & MB: 0.0066 & MB: 0.0045 & MB: 0.0120 \\
\hline & & UB: 0.0098 & UB: 0.0065 & UB: 0.0240 \\
\hline \multirow{3}{*}{$\begin{array}{l}\text { Mixed cereals } \\
\text { for infants }\end{array}$} & \multirow{3}{*}{73} & LB: 0.0068 & LB: 0.0000 & LB: 0.0180 \\
\hline & & MB: 0.0102 & MB: 0.0100 & MB: 0.0180 \\
\hline & & UB: 0.0137 & UB: 0.0117 & UB: 0.0240 \\
\hline \multirow{3}{*}{$\begin{array}{l}\text { Biscuits } \\
\text { for infants }\end{array}$} & \multirow{3}{*}{49} & LB: 0.0067 & LB: 0.0000 & LB: 0.0164 \\
\hline & & MB: 0.0087 & MB: 0.0045 & MB: 0.0180 \\
\hline & & UB: 0.0107 & UB: 0.0060 & UB: 0.0182 \\
\hline \multirow{3}{*}{$\begin{array}{l}\text { Vegetable } \\
\text { meals } \\
\text { ready-to-eat }\end{array}$} & \multirow{3}{*}{49} & LB: 0.0040 & LB: 0.0000 & LB: 0.0130 \\
\hline & & MB: 0.0061 & MB: 0.0050 & MB: 0.0144 \\
\hline & & UB: 0.0082 & UB: 0.0060 & UB: 0.0144 \\
\hline \multirow{3}{*}{$\begin{array}{l}\text { Vegetable- } \\
\text { meat meals } \\
\text { ready-to-eat }\end{array}$} & \multirow{3}{*}{62} & LB: 0.0029 & LB: 0.0000 & LB: 0.0117 \\
\hline & & MB: 0.0058 & MB: 0.0028 & MB: 0.0175 \\
\hline & & UB: 0.0087 & UB: 0.0050 & UB: 0.0198 \\
\hline \multirow{3}{*}{$\begin{array}{l}\text { Fish-based } \\
\text { infant foods } \\
\text { ready-to-eat }\end{array}$} & \multirow{3}{*}{47} & LB: 0.0663 & LB: 0.0530 & LB: 0.1781 \\
\hline & & MB: 0.0666 & MB: 0.0530 & MB: 0.1781 \\
\hline & & UB: 0.0670 & UB: 0.0530 & UB: 0.1781 \\
\hline \multirow{3}{*}{$\begin{array}{l}\text { Fruits desserts } \\
\text { for infants }\end{array}$} & \multirow{3}{*}{43} & LB: 0.0032 & LB: 0.0000 & LB: 0.0136 \\
\hline & & MB: 0.0055 & MB: 0.0025 & MB: 0.0166 \\
\hline & & UB: 0.0079 & UB: 0.0050 & UB: 0.0166 \\
\hline \multirow{3}{*}{$\begin{array}{l}\text { Juices } \\
\text { for infants }\end{array}$} & & LB: 0.0037 & LB: 0.0000 & LB: 0.0130 \\
\hline & 69 & MB: 0.0058 & MB: 0.0030 & MB: 0.0156 \\
\hline & & UB: 0.0078 & UB: 0.0050 & UB: 0.0156 \\
\hline & & LB: 0.0036 & LB: 0.0000 & LB: 0.0120 \\
\hline Herbal teas & 45 & MB: 0.0071 & MB: 0.0025 & MB: 0.0180 \\
\hline & & UB: 0.0107 & UB: 0.0050 & UB: 0.0200 \\
\hline Herbal and & & LB: 0.0013 & LB: 0.0000 & LB: 0.0033 \\
\hline & 60 & MB: 0.0045 & MB: 0.0025 & MB: 0.0100 \\
\hline & & UB: 0.0077 & UB: 0.0050 & UB: 0.0200 \\
\hline
\end{tabular}

$\mathrm{n}$ - number of samples, LB - lower bound, MB - middle bound, UB - upper bound, $\mathrm{P} 90-90^{\text {th }}$ percentile 
Table 4. Mercury content in different types of commercially available infant foods in the Polish market $(\mathrm{mg} / \mathrm{kg})$.

\begin{tabular}{|c|c|c|c|c|}
\hline \multirow{2}{*}{ Product } & \multicolumn{4}{|c|}{$\mathrm{Hg}$} \\
\hline & $\mathrm{n}$ & Mean & Median & P90 \\
\hline \multirow{3}{*}{$\begin{array}{l}\text { Infant } \\
\text { formula, } \\
\text { follow-on } \\
\text { formula }\end{array}$} & \multirow{3}{*}{113} & LB: 0.0004 & LB: 0.0000 & LB: 0.0010 \\
\hline & & MB: 0.0007 & MB: 0.0005 & MB: 0.0010 \\
\hline & & UB: 0.0010 & UB: 0.0010 & UB: 0.0020 \\
\hline \multirow{3}{*}{$\begin{array}{l}\text { Milk and } \\
\text { rice-based } \\
\text { products }\end{array}$} & \multirow{3}{*}{61} & LB: 0.0004 & LB: 0.0000 & LB: 0.0012 \\
\hline & & MB: 0.0006 & MB: 0.0005 & MB: 0.0012 \\
\hline & & UB: 0.0009 & UB: 0.0010 & UB: 0.0019 \\
\hline \multirow{3}{*}{$\begin{array}{c}\text { Infant formula } \\
\text { soya-based }\end{array}$} & \multirow{3}{*}{22} & LB: 0.0009 & LB: 0.0005 & LB: 0.0020 \\
\hline & & MB: 0.0009 & MB: 0.0005 & MB: 0.0020 \\
\hline & & UB: 0.0013 & UB: 0.0010 & UB: 0.0020 \\
\hline \multirow{3}{*}{$\begin{array}{l}\text { Rice gruel } \\
\text { for infants }\end{array}$} & \multirow{3}{*}{180} & LB: 0.0005 & LB: 0.0000 & LB: 0.0020 \\
\hline & & MB: 0.0009 & MB: 0.0006 & MB: 0.0020 \\
\hline & & UB: 0.0013 & UB: 0.0010 & UB: 0.0020 \\
\hline \multirow{3}{*}{$\begin{array}{l}\text { Wheat gruel } \\
\text { for infants }\end{array}$} & \multirow{3}{*}{76} & LB: 0.0002 & LB: 0.0000 & LB: 0.0010 \\
\hline & & MB: 0.0006 & MB: 0.0005 & MB: 0.0010 \\
\hline & & UB: 0.0009 & UB: 0.0010 & UB: 0.0011 \\
\hline \multirow{3}{*}{$\begin{array}{l}\text { Mixed cereals } \\
\text { for infants }\end{array}$} & \multirow{3}{*}{66} & LB: 0.0005 & LB: 0.0000 & LB: 0.0015 \\
\hline & & MB: 0.0008 & MB: 0.0005 & MB: 0.0015 \\
\hline & & UB: 0.0011 & UB: 0.0010 & UB: 0.0017 \\
\hline \multirow{3}{*}{$\begin{array}{l}\text { Biscuits } \\
\text { for infants }\end{array}$} & \multirow{3}{*}{48} & LB: 0.0005 & LB: 0.0000 & LB: 0.0018 \\
\hline & & MB: 0.0007 & MB: 0.0005 & MB: 0.0018 \\
\hline & & UB: 0.0010 & UB: 0.0010 & UB: 0.0018 \\
\hline \multirow{3}{*}{$\begin{array}{l}\text { Vegetable } \\
\text { meals } \\
\text { ready-to-eat }\end{array}$} & \multirow{3}{*}{49} & LB: 0.0003 & LB: 0.0000 & LB: 0.0010 \\
\hline & & MB: 0.0006 & MB: 0.0005 & MB: 0.0010 \\
\hline & & UB: 0.0009 & UB: 0.0010 & UB: 0.0010 \\
\hline \multirow{3}{*}{$\begin{array}{l}\text { Vegetable- } \\
\text { meat meals } \\
\text { ready-to-eat }\end{array}$} & \multirow{3}{*}{62} & LB: 0.0006 & LB: 0.0000 & LB: 0.0014 \\
\hline & & MB: 0.0009 & MB: 0.0005 & MB: 0.0014 \\
\hline & & UB: 0.0012 & UB: 0.0010 & UB: 0.0020 \\
\hline \multirow{3}{*}{$\begin{array}{l}\text { Fish-based } \\
\text { infant foods } \\
\text { ready-to-eat }\end{array}$} & \multirow{3}{*}{47} & LB: 0.0063 & LB: 0.0056 & LB: 0.0127 \\
\hline & & MB: 0.0063 & MB: 0.0056 & MB: 0.0127 \\
\hline & & UB: 0.0064 & UB: 0.0056 & UB: 0.0127 \\
\hline \multirow{3}{*}{$\begin{array}{c}\text { Fruits desserts } \\
\text { for infants }\end{array}$} & \multirow{3}{*}{41} & LB: 0.0003 & LB: 0.0000 & LB: 0.0010 \\
\hline & & MB: 0.0005 & MB: 0.0005 & MB: 0.0010 \\
\hline & & UB: 0.0008 & UB: 0.0010 & UB: 0.0011 \\
\hline \multirow{3}{*}{$\begin{array}{l}\text { Juices } \\
\text { for infants }\end{array}$} & & LB: 0.0002 & LB: 0.0000 & LB: 0.0010 \\
\hline & 68 & MB: 0.0004 & MB: 0.0003 & MB: 0.0010 \\
\hline & & UB: 0.0007 & UB: 0.0005 & UB: 0.0010 \\
\hline & & LB: 0.0004 & LB: 0.0000 & LB: 0.0010 \\
\hline Herbal teas & 44 & MB: 0.0006 & MB: 0.0005 & MB: 0.0010 \\
\hline & & UB: 0.0008 & UB: 0.0010 & UB: 0.0010 \\
\hline Herbal and & & LB: 0.0002 & LB: 0.0000 & LB: 0.0009 \\
\hline & 60 & MB: 0.0005 & MB: 0.0005 & MB: 0.0009 \\
\hline & & UB: 0.0008 & UB: 0.0010 & UB: 0.0010 \\
\hline
\end{tabular}

$\mathrm{n}$ - number of samples, LB - lower bound, MB - middle bound, UB - upper bound, $\mathrm{P} 90-90^{\text {th }}$ percentile

\section{Lead Contamination}

Content of lead, taking into consideration mean MB contamination, was lower as compared with results obtained during studies performed in Poland in 2004-08 $[20,21]$. Mean lead content in tested samples of infant formula and follow-on formula was $0.005 \mathrm{mg} / \mathrm{kg}, 90^{\text {th }}$ percentile MB were below $0.010 \mathrm{mg} / \mathrm{kg}$. The highest value of lead was detected in a sample of powdered infant formula at $0.056 \mathrm{mg} / \mathrm{kg}$, but after reconstitution did not exceed the permitted maximum level $0.020 \mathrm{mg} / \mathrm{kg}$ set by Commission Regulation No.1881/2006, as amended. Obtained results for infant formulas were just slightly higher than those reported in other European countries. According to EFSA, the mean (MB) was in the range $0.003-0.004 \mathrm{mg} / \mathrm{kg}$ [22] - significantly lower than reported in Pakistan for commercially available milk-based and soya-based infant formula (mean values 0.0287-0.097 $\mathrm{mg} / \mathrm{kg}$ and $0.098-0.119 \mathrm{mg} / \mathrm{kg}$, respectively) [23].

The survey conducted by the Food Standards Agency in the U.K. indicates lower contamination of infant formulae as compared with results obtained in Poland (mean was in the range $0.002-0.003 \mathrm{mg} / \mathrm{kg}$ ). Infant formulae based on cow's milk proteins contribute less to lead intake than soya milk formulae, the mean (MB) is $0.006 \mathrm{mg} / \mathrm{kg}$ (the highest reported result was $0.025 \mathrm{mg} / \mathrm{kg}$ for follow-on formula the product as sold) and was comparable with results reported in the U.K. for this group of products (mean 0.007 $\mathrm{mg} / \mathrm{kg}$ ) [24]. Contents of lead in milk and rice-based products were just slightly lower as compared with infant formulas. Lead levels in the products sampled in Poland were lower than those reported in Germany [2]. Detected rice gruel contamination was significantly higher than for other cereal-based products for infants. Studies conducted in Sweden confirm this dependence [7]. Average lead content in rice gruel was $0.008 \mathrm{mg} / \mathrm{kg}$, as compared with mean value $0.006 \mathrm{mg} / \mathrm{kg}$ for mixed cereals and wheat gruel. Highest lead content at $0.120 \mathrm{mg} / \mathrm{kg}$ was reported in pure rice gruel.

Mean lead content in biscuits was $0.012 \mathrm{mg} / \mathrm{kg}$, median $0.005 \mathrm{mg} / \mathrm{kg}$, and $90^{\text {th }}$ percentile was below $0.030 \mathrm{mg} / \mathrm{kg}$. Reported lead contamination of biscuits in Poland was significantly lower than in other EU countries with mean (MB) $0.019 \mathrm{mg} / \mathrm{kg}$ [22], which is comparable with contamination found in the U.K. [24].

Lead content in ready-to-eat solid infant foods, i.e., vegetable meals, meals based on vegetables and meat, fishbased infant foods, and fruits, as well as for herbs and fruit teas, approx. $0.007 \mathrm{mg} / \mathrm{kg}$ (mean) did not differ significantly and was comparable with contamination reported in Germany for solid baby foods [2]. Lead contamination of vegetable meals and meat and vegetable-based meals, as well as fruit purée, was slightly lower from that reported by other EU Member States [22, 24].

Mean MB lead contamination in herbal teas and juices for infants was comparable and did not create any health hazard. 


\section{Cadmium Contamination}

Contamination of vegetable meals, vegetable and meat meals, biscuits, and fish-based infant foods with cadmium was in the range $0.007-0.010 \mathrm{mg} / \mathrm{kg}$ (mean MB), median $0.006-0.008 \mathrm{mg} / \mathrm{kg}$, and $90^{\text {th }}$ percentile $0.010-0.017 \mathrm{mg} / \mathrm{kg}$, and was comparable with results collected by EFSA from EU Member States [25]. The highest content of cadmium in vegetable meals was $0.044 \mathrm{mg} / \mathrm{kg}$ in a meal based on spinach and potatoes. The survey conducted in the U.K. showed higher cadmium content in aforementioned groups of products except fish-based meals, whose contamination was similar to that reported in Poland [24].

Mean cadmium content in infant formulae and followon formulae was comparable (mean MB $0.0012 \mathrm{mg} / \mathrm{kg}$ ) with milk- and rice-based products (mean MB 0.0016 $\mathrm{mg} / \mathrm{kg}$ ). Infant formulae based on cow's milk proteins contribute less to cadmium intake than soya milk formulae. The mean in this case is $0.0034 \mathrm{mg} / \mathrm{kg}$ and is lower than that reported by other European countries [24]. The highest cadmium content in a soya-based infant formula sample was $0.012 \mathrm{mg} / \mathrm{kg}$ but did not exceed the permitted maximum level of $0.020 \mathrm{mg} / \mathrm{kg}$ set by Commission Regulation No. 488/2014, amending Regulation (EC) No. 1881/2006. Cadmium contamination of infant formulas was lower as compared with results from previous studies conducted in Poland [26]. Cereal-based products can contribute significantly to dietary cadmium intake. Mean cadmium levels in tested samples of wheat gruel were $0.0047 \mathrm{mg} / \mathrm{kg}$, for rice gruel $0.0041 \mathrm{mg} / \mathrm{kg}$, and $90^{\text {th }}$ percentile value was 0.009 $\mathrm{mg} / \mathrm{kg}$ and $0.012 \mathrm{mg} / \mathrm{kg}$, respectively. The highest value was reported in pure rice gruel intended for consumption by 4-month-old infants: mean $0.0490 \mathrm{mg} / \mathrm{kg}$ and was higher than maximum level of $0.040 \mathrm{mg} / \mathrm{kg}$ set by the Commission Regulation No. 488/2014. Cadmium levels in mixed cereal samples were lower, and mean MB was $0.0035 \mathrm{mg} / \mathrm{kg}$.

The results reported in Pakistan [7, 27, 28] indicate higher contamination of cereal-based infant foods than in Poland. Content of cadmium in other infant foods, i.e., juices, fruit-based desserts, herbal teas, and teas with the addition of fruits was lower compared with the above-mentioned infant foods. The mean value ranged from 0.0006 $\mathrm{mg} / \mathrm{kg}$ for herbal teas to $0.0018 \mathrm{mg} / \mathrm{kg}$ for fruit desserts.

\section{Arsenic Contamination}

Arsenic content in rice gruel and fish-based infant foods was significant: mean MB for rice gruel was $0.060 \mathrm{mg} / \mathrm{kg}$, median $0.045 \mathrm{mg} / \mathrm{kg}$, and $90^{\text {th }}$ percentile value 0.140 $\mathrm{mg} / \mathrm{kg}$; for fish- based infant foods $0.067 \mathrm{mg} / \mathrm{kg}$, median $0.053 \mathrm{mg} / \mathrm{kg}$, and $90 \%$ value $0.178 \mathrm{mg} / \mathrm{kg}$. Highest content of arsenic for these two groups of products was reported in the sample of rice gruel at $0.297 \mathrm{mg} / \mathrm{kg}$ and in cod fish with vegetables dinner sample at $0.195 \mathrm{mg} / \mathrm{kg}$. Arsenic contamination of fish-based infant foods in Poland was lower than that reported in Norway (mean $1.10 \mathrm{mg} / \mathrm{kg}$ ) [10], Spain (mean $0.619 \mathrm{mg} / \mathrm{kg}$ [29]), and the U.K. (mean 0.145 $\mathrm{mg} / \mathrm{kg}$ ) [24]. The surveys conducted in Spain and the U.K. [24] also indicated higher contamination of pure infant rice with total arsenic than reported in Poland; mean was 0.126 $\mathrm{mg} / \mathrm{kg}$ (fresh weight) and $0.183 \mathrm{mg} / \mathrm{kg}$, respectively. Other Spanish data show the mean values of arsenic contamination at $0.089 \mathrm{mg} / \mathrm{kg}$ for pure rice conventional products and at $0.168 \mathrm{mg} / \mathrm{kg}$ for organic products produced using organic farming methods [30]. Similarly higher results than in Poland were observed in other EU member states, mean LB was $0.1496 \mathrm{mg} / \mathrm{kg}$ and UB $0.1575 \mathrm{mg} / \mathrm{kg}$ [15]. A Swedish investigation showed lower contamination of rice than in Poland (mean contents of arsenic in infant products based on rice ranged from $0.017 \mathrm{mg} / \mathrm{kg}$ to $0.033 \mathrm{mg} / \mathrm{kg}$ [7].

Arsenic is present in rice and rice products mainly as an inorganic form being more toxic than organic compounds; intake of inorganic arsenic by infants can create a health hazard [29, 31, 32]. For infants, rice products can contribute significantly to total exposure to arsenic, even up to $31 \%$ [15]. Content of arsenic in other infant foods was low and did not create any health hazard; mean ranged from 0.004 $\mathrm{mg} / \mathrm{kg}$ to $0.012 \mathrm{mg} / \mathrm{kg}$; median $0.003-0.010 \mathrm{mg} / \mathrm{kg}$; and $90 \%$ value $0.010-0.026 \mathrm{mg} / \mathrm{kg}$. Reported arsenic levels in domestic investigations were lower than results obtained in other EU member states [15].

\section{Mercury Contamination}

The reported mean mercury contents were low and did not pose a health hazard except for fish-based infant foods. Fish and fishery products are the main source of methylmercury ( $\mathrm{MeHg}$ ) exposure in humans, especially in infants and children [17, 33-35]. Fish-containing dishes had the highest levels of mercury. The mean contents $(\mathrm{MB})$ for this group of products were $0.0063 \mathrm{mg} / \mathrm{kg}$ (median 0.0056 $\mathrm{mg} / \mathrm{kg}, 90 \%$ value $-0.0127 \mathrm{mg} / \mathrm{kg}$ ), which is comparable with results reported in Norway [10]. Similar values in baby food containing fish were reported in other EU member states $[24,35]$. Contamination of other products with mercury was significantly lower and ranged from 0.0004 $\mathrm{mg} / \mathrm{kg}$ for juices to $0.0009 \mathrm{mg} / \mathrm{kg}$ for rice gruel. The highest value of mercury was found in a vegetable and fishbased infant dinner sample, at $0.0185 \mathrm{mg} / \mathrm{kg}$.

\section{Estimation of Intake of Toxic Elements}

The calculation of dietary exposure of infants was performed based on the artificial feeding scheme of infants established under The Mother and Child Institute in Poland for 3-month-old infants weighing $6 \mathrm{~kg}$ who fully fed on infant formulae based on milk or soya, and 12-month-old infants weighing $10 \mathrm{~kg}$, consuming additionally puréed infant foods and cereals-based formulae [36]. Additionally, manufacturers' feeding recommendations as labeled on the products (mainly formulas) were taken into consideration to assess dietary exposure of infants. Health risk assessment was performed taking into account current reference values for elements including the verification introduced recently by EFSA and JECFA. To assess the dietary exposure to noxious elements from commercially available infant food mean and high contamination levels (P90), middle bound 
(MB), and upper bound (UB) values were taken into account. This assessment assumes an infant diet consisting exclusively of commercial baby food.

The calculated intake is based on the obtained analytical results of heavy metals contamination of undiluted, commercial baby foods (as sold).

However, be aware that the real intake of elements under study by infants is higher, primarily due to contamination of water used to reconstitute infant formulas and other dried foods before feeding, in accordance with manufacturers' recommendations.

In the case of lead and cadmium the calculated intake of metals is presented additionally, including water contamination.

Intake of elements noxious to health by 3- and 12 month-old infants based on middle bound (MB) contamination level and mean consumption is presented in Figs. 1-5. Overall intake of heavy metals by 3- and 12-month-old infants with investigated infant food (without water), based on additional assumptions $\left(90^{\text {th }}\right.$ percentile $\mathrm{MB}$ and UB as well as mean MB and UB) is presented in Figs. 6 and 7.

\section{Intake of Lead}

The calculated mean intake of lead with infant foods as a result of reported contamination of products as sold, based on MB values of contamination, was by 12 -monthold infants at $0.53 \mu \mathrm{g} / \mathrm{kg}$ b.w. per day, and $0.09 \mu \mathrm{g} / \mathrm{kg}$ b.w. per day by 3-month-old infants. Lead exposure based on $90^{\text {th }}$ percentile MB assumption ranges from $0.17 \mu \mathrm{g} / \mathrm{kg}$ b.w. per day (infants) to $1.05 \mu \mathrm{g} / \mathrm{kg}$ b.w. per day (1 year olds), and at the $90^{\text {th }}$ percentile contamination, UB assumption may even be $0.2 \mu \mathrm{g} / \mathrm{kg}$ b.w. for 3-month-old infants and 1.2 $\mu \mathrm{g} / \mathrm{kg}$ b.w. for 12 -month-olds. Even though this assumption does not take into account water used for formulae reconstitution, intake of lead in the aforementioned case is 2.5 times higher than $\mathrm{BMDL}_{01}$ value for 12-month-old infants and $42 \%$ of $\mathrm{BMDL}_{01}$ value for infants weighing $6 \mathrm{~kg}$. Mean intake of lead (based on mean, middle bound levels of infant food contamination) by 12 -month-old infants is approximately equal to the $\mathrm{BMDL}_{01}$ value Benchmark dose lower confidence limit causing a $1 \%$ increase of neurodevelopment effects in children. Lead intake is highest with

$\square$ 12-month-old infants (10 kg) $\square$ 3-month-old infants (6 kg)

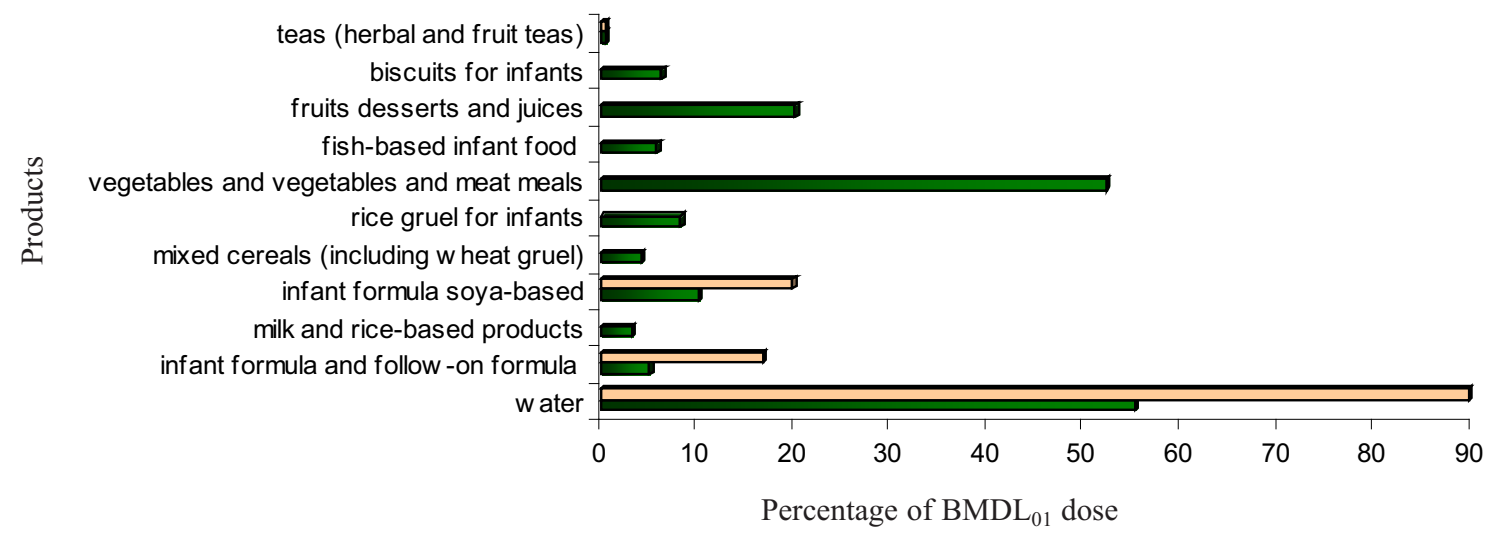

Fig. 1. Lead intake by infants (3 and 12 months old) based on middle-bound (MB) contamination level.

$\square$ 12-month-old infants (10 kg) $\square$ 3-month-old infants (6 kg)

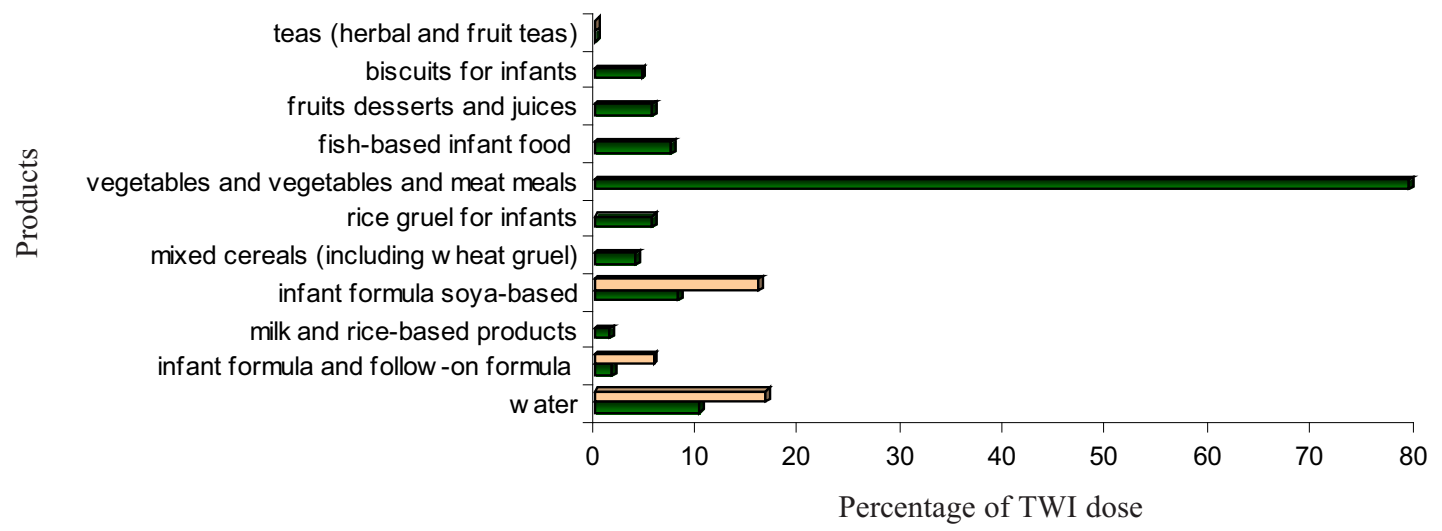

Fig. 2. Cadmium intake by infants (3 and 12 months old) based on middle-bound (MB) contamination level. 
vegetable products, products with meat and vegetables, and fruit and juices. Mean intake by 3-month-old infants fed on exclusively milk- and soy-based infant formulae would cover $17 \%$ of $\mathrm{BMDL}_{01}$ value.
Taking into account the contamination of water necessary for product reconstitution before feeding due to manufacturer's recommendations (mean tap water contamination in Poland), [37] mean total lead intake by 12 -month-olds

12-month-old infants (10 kg) $\square$ 3-month-old infants $(6 \mathrm{~kg})$

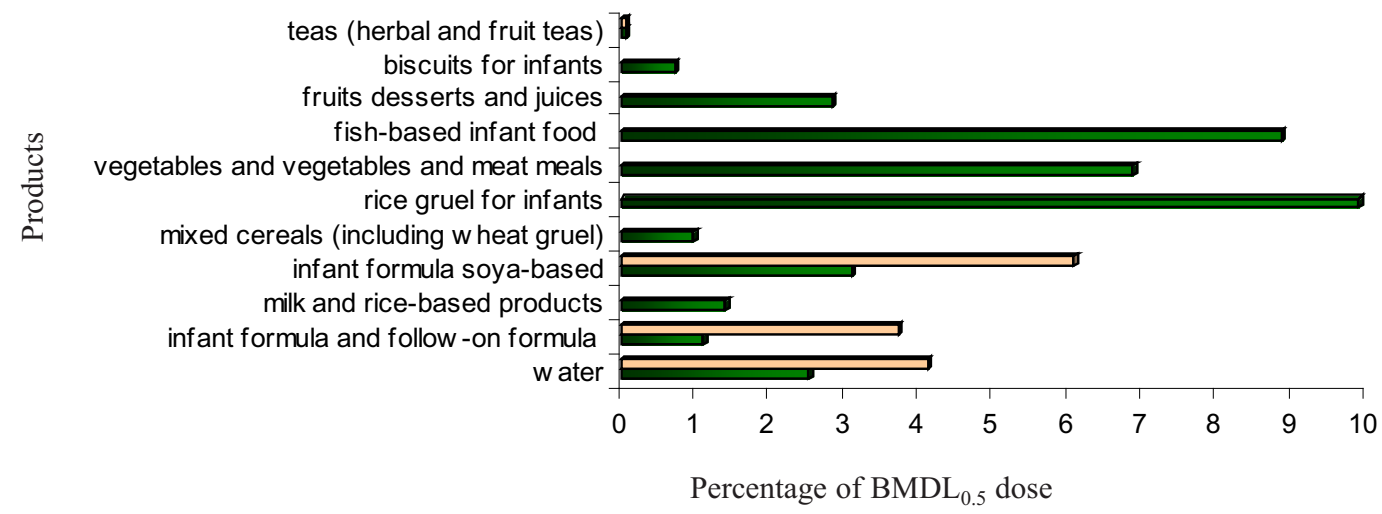

Fig. 3. Arsenic intake by infants ( 3 and 12 months old) based on middle-bound (MB) contamination level.

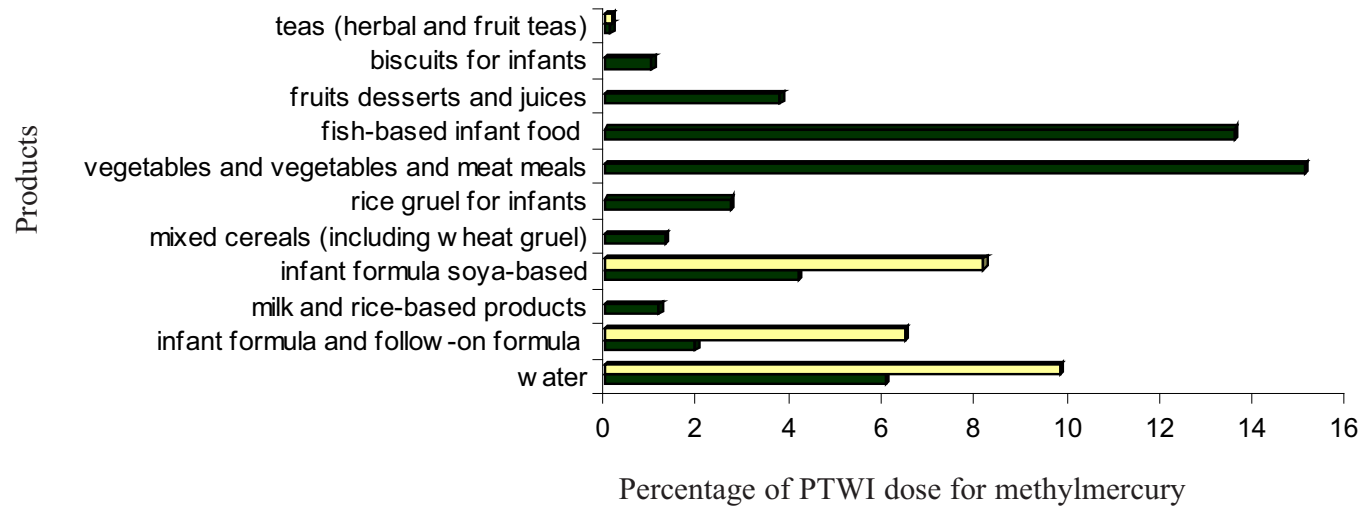

Fig. 4. Methylmercury intake by infants (3 and 12 months old) based on middle-bound (MB) contamination level.

— 12-month-old infants (10 kg) $\square$ 3-month-old infants (6 kg)

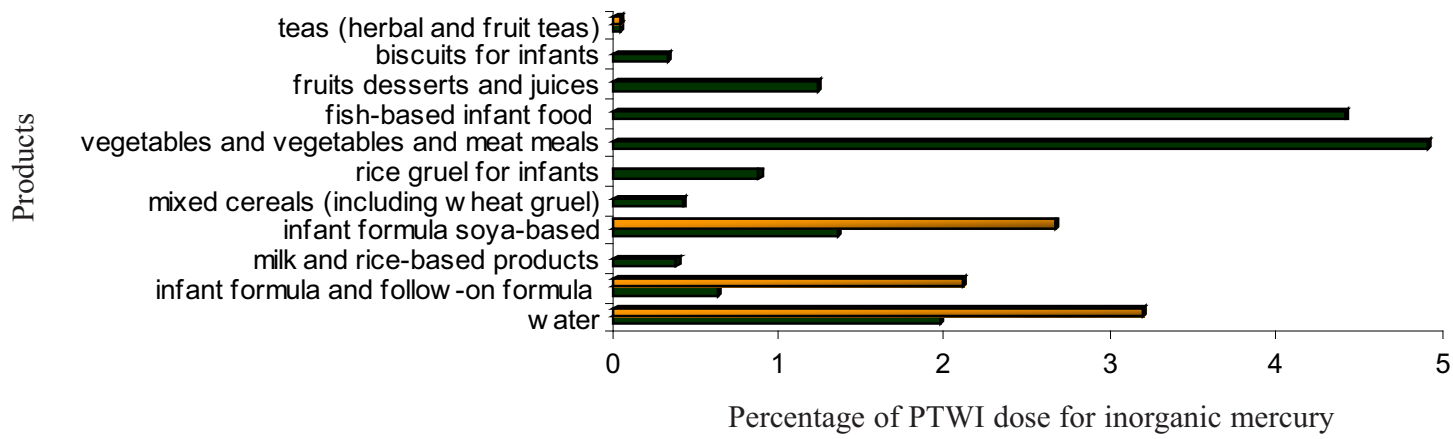

Fig. 5. Inorganic mercury intake by infants (3 and 12 months old) based on middle-bound (MB) contamination level. 
would rise to $0.81 \mu \mathrm{g} / \mathrm{kg}$ b.w. per day (1.6 times higher than $\mathrm{BMDL}_{01}$ ) and by 3 -month-olds to $0.54 \mu \mathrm{g} / \mathrm{kg}$ b.w. per day (equal approx. the $\mathrm{BMDL}_{01}$ value). The margin of exposure (MOE) calculated for 3-month-old infants and 1-year-olds fed on infant and follow-on formulae and other commercially available baby food (prepared for feeding with the use of tap water) ranges between 0.9 for infants to 0.6 for 1-year-olds.

The actual lead level of tap water in Poland could cause a high increase of intake of this toxic element by infants: about $60 \%$ of $\mathrm{BMDL}_{01}$ (12-month-old infants) $-90 \%$ of $\mathrm{BMDL}_{01}$ (3-month-old infants).

The assessed maximum daily intake of lead for 3month-olds is significantly lower than the maximum intake of lead from infant formula in the USA, at $3.4 \mu \mathrm{g} /$ day [6]. The assessed upper bound mean exposure values for infants were as follows: 0.13 and $0.69 \mu \mathrm{g} / \mathrm{kg}$ b.w. per day (without water). Estimated average exposure for infants in EU fed with ready-to-consume infant formula was $0.63 \mu \mathrm{g} / \mathrm{kg}$ b.w. per day, calculated based on upper-bound assumptions [12], and was similar to that reported in Poland.

\section{Intake of Cadmium}

Mean intake of cadmium resulted only from products as sold contamination, ranges from $6 \%$ of the TWI established by EFSA for 3-month-old infants $(0.02 \mu \mathrm{g} / \mathrm{kg}$ b.w. per day) to $110 \%$ TWI for 12 -month-old infants $(0.39 \mu \mathrm{g} / \mathrm{kg}$ b.w. per day), which represents $2.5-47 \%$ of the PTMI value established by JECFA. Water used for product reconstitution increases the cadmium intake to $23-121 \%$ of the TWI (about $10-52 \%$ of PTMI), respectively. The highest intake of cadmium was assessed with vegetables and meat and vegetable products at $2.8 \mu \mathrm{g} / \mathrm{kg}$ b.w. per day. According to EFSA opinion, dominating contributors to cadmium exposure of infants are ready-to-eat meals, powder infant formulae, and cereal-based food for infants [25].

Intake of cadmium may be even higher for children allergic to cow milk proteins who consume soya products instead - 16\% (with water $33 \%$ of TWI) for 3-month-old infants, which corresponds to $7 \%$ (14\%) of the PTMI.

Cadmium exposure based on $90^{\text {th }}$ percentile MB assumption without considering water ranges from $0.05 \mu \mathrm{g} / \mathrm{kg}$ b.w.

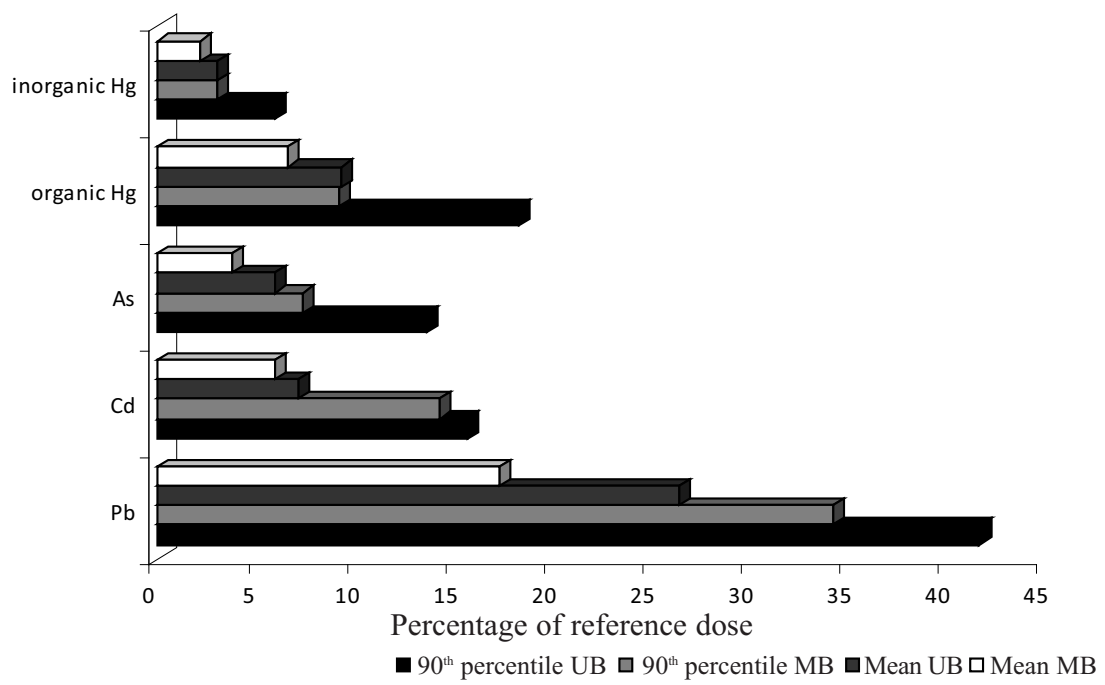

Fig. 6. Intake of elements noxious to health with investigated infant food by 3 -month-old infants (without water).

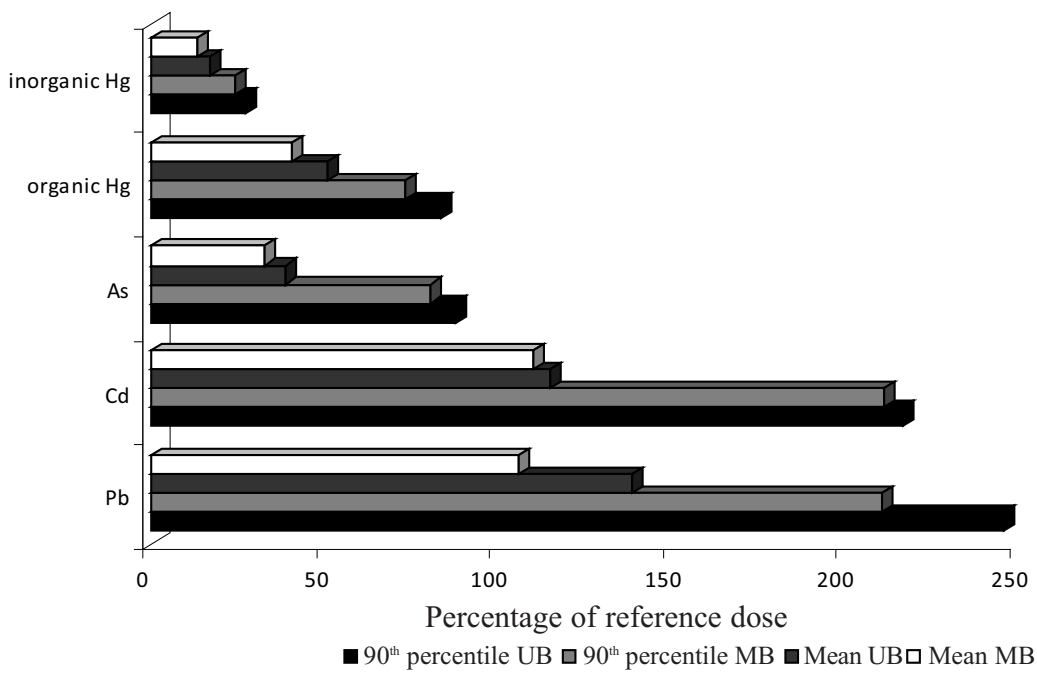

Fig. 7. Intake of elements noxious to health with investigated infant food by 12 -month-old infants (without water). 
per day (14\% TWI) for infants to $0.76 \mu \mathrm{g} / \mathrm{kg}$ b.w. per day for 1-year-olds (2.1 times higher than the TWI value).

At the $90^{\text {th }}$ percentile contamination levels based on UB assumption of contamination, cadmium intake by different age groups of infants ranges from 0.06 to $0.77 \mu \mathrm{g} / \mathrm{kg} \mathrm{b.w}$. per day and may exceed the TWI by about 2.2-times in relation to 1-year-old children (93\% PTMI value). Exposure of 3-month-old infants at these levels of contamination was assessed to be $16 \%$ of TWI value (7\% PTMI).

UB mean exposure values were $0.03 \mu \mathrm{g} / \mathrm{kg}$ b.w. per day for infants (7\% TWI and 3\% PTMI) and $0.41 \mu \mathrm{g} / \mathrm{kg}$ b.w. per day for 1-year-old children (115\% of TWI value and $49 \%$ PTMI), and are lower than the exposure calculated by EFSA for infants $(<1$ year) at $3.5 \mu \mathrm{g} / \mathrm{kg}$ b.w. per day [25].

\section{Intake of Arsenic}

Intake of arsenic does not exceed tolerable doses (approx. 33\% of $\mathrm{BMDL}_{0.5}$ value for 12-month-old infants and $4 \%$ for 3 -month-old infants, which corresponds to exposure values 0.99 and $0.11 \mu \mathrm{g} / \mathrm{kg}$ b.w. per day - middle bound). Arsenic exposure based on $90^{\text {th }}$ percentile $\mathrm{MB}$ assumption ranges from $0.22 \mu \mathrm{g} / \mathrm{kg}$ b.w. per day for 3month-old infants to $2.4 \mu \mathrm{g} / \mathrm{kg}$ b.w. per day for 1-year-olds (7-81\% $\mathrm{BMDL}_{0.5}$ value). At the $90^{\text {th }}$ percentile UB level of contamination intake by 12 -month-olds is equal approximately to the $\mathrm{BMDL}_{0.5}$ value $(2.6 \mu \mathrm{g} / \mathrm{kg}$ b.w. per day) and corresponds with daily exposure estimated in the United States for infants [15]. The assessed MOE depending on contamination levels ranges from 1.1 for 12-month olds to 7.3 for 3-month olds. Estimated intake of arsenic by infants weighing $6 \mathrm{~kg}$, at this level of contamination (UB, $90^{\text {th }}$ percentile), was $0.4 \mu \mathrm{g} / \mathrm{kg}$ b.w. per day (14\% $\mathrm{BMDL}_{0.5}$ value). The highest intake of arsenic was assessed for rice gruel, fish-based baby foods, and vegetable and meat meals and infant formulas. Calculated mean upper-bound exposure of arsenic by 3-month-old infants was estimated to be 0.2 $\mu \mathrm{g} / \mathrm{kg}$ b.w. per day (6\% BMDL $\mathrm{B}_{0.5}$ value), and $1.2 \mu \mathrm{g} / \mathrm{kg}$ b.w. per day $\left(39 \% \mathrm{BMDL}_{0.5}\right)$ by 12 -month-olds.

The contamination of tap water in Poland with arsenic is relatively less essential than with lead and cadmium and causes an increase of arsenic intake about $3-4 \%$ of $\mathrm{BMDL}_{0.5}$.

\section{Intake of Mercury}

Intake of mercury, assuming that total mercury is represented by its organic compounds (methylmercury - "worst case scenario") does not exceed tolerable doses $(1.3 \mu \mathrm{g} / \mathrm{kg}$ b.w. per week). Based on mean MB concentration of mercury in infants food, the dietary exposure to more toxic organic mercury from the investigated products ranged from $0.01 \mu \mathrm{g} / \mathrm{kg}$ b.w. per day for 3-month-old infants to 0.08 $\mu \mathrm{g} / \mathrm{kg}$ b.w. per day for 12-month-olds (7-41\% TWI value for organic mercury). Calculated mean MB intake of organic compounds of mercury with fish products in Poland by 1year-old children was $0.025 \mu \mathrm{g} / \mathrm{kg}$ b.w. per day (14\% TWI value for organic mercury), and is lower than that reported in Finland (mean: 0.028-0.032 $\mu \mathrm{g} / \mathrm{kg}$ b.w. per day) [33].
Calculated intake of mercury based on $90^{\text {th }}$ percentile of contamination and MB assumption ranges from $0.02 \mu \mathrm{g} / \mathrm{kg}$ b.w. per day for 3-month-old infants to $0.14 \mu \mathrm{g} / \mathrm{kg}$ b.w. per day for 1-year-olds $(9-73 \%$ TWI value for organic mercury). Mercury exposure based on $90^{\text {th }}$ percentile UB assumption of contamination ranges from 0.03 for infants to $0.15 \mu \mathrm{g} / \mathrm{kg}$ b.w. per day for 1 -year-olds. Dietary intake in this case approximately equals the TWI for methylmercury (83\%) for 12 -month-olds and $18 \%$ of TWI value for 3 month-old infants.

Assuming that all the mercury represented in the investigated samples is inorganic (which is the more appropriate scenario), intake of mercury based on mean contamination and middle bound assumption corresponds to $2 \%$ of PTWI value adopted by JECFA ( $4.0 \mu \mathrm{g} / \mathrm{kg}$ b.w.) for 3-month-old infants and $13 \%$ for 12 -month-olds. Taking into account $90^{\text {th }}$ percentile middle bound assumption intake of mercury (as inorganic) will be in the range 3-24\%, respectively. At the $90^{\text {th }}$ percentile contamination of mercury, UB assumption intake ranges between $6 \%$ and $27 \%$ of PTWI value for 3-month-old infants and 1-year-olds. The use of tap water for commercial products reconstitution causes increased methylmercury intake of about $6-10 \%$ of TWI (about $2-3 \%$ of TWI for inorganic mercury).

This study indicates that fish-based infant food as well as vegetables, vegetable and meat, fruit desserts, and juices dominate contributors to mercury intake by infants. Readyto-eat fruit-based foods also contributed the most to inorganic mercury exposure in French studies [38].

\section{Conclusions}

The results of these studies indicate that the heavy metals levels in infant food from Polish markets are comparable with contamination in other countries. However, intake of these elements with food by 12 -month-olds in some cases may exceed reference values established by EFSA and JECFA. Even though the levels of lead were lower than mean tap water contamination in all products under study, cadmium and lead can pose a health hazard for infants.

Drinking tap water used to prepare dishes may add significantly to heavy metals intake, especially for 3-monthold infants whose diet consists mainly of infant formulae, mainly in the case of lead and cadmium.

Another important issue is contamination of rice products with arsenic, which seems to be significant.

Furthermore, diets with rice and fish-based products contributed most to dietary arsenic intake. Fish-based infant foods as well as ready-to-eat meals based on vegetables and meat or only vegetables can also contribute to mercury intake.

It is important to mitigate health risks by imposing a set of maximum permissible levels for all toxic elements in infant foods into the applicable legislation, especially in foodstuffs that characterise higher toxic metals contamination. Manufacturers of infant foods should ensure the quality of their products by selecting raw materials. 


\section{Acknowledgements}

The authors would like to thank the staff of SanitaryEpidemiological Stations for their assistance and cooperation in this program.

\section{References}

1. PATRIARCA M., MENDITTO A., ROSSI B., LYON T.D.B., FELL G.S. Environmental exposure to metals of newborns, infants and young children. Microchem. J. 67, 351, 2000

2. PANDELOVA M., LOPEZ W. L., MICHALKE B., SCHRAMM K.W. Ca, Cd Cu, Fe, Hg, Mn, Ni, Pb, Se and Zn contents in baby foods from the EU market: Comparison of assessed infant intakes with the present safety limits for minerals and trace elements. J. Food Compos. Anal. 27, 120, 2012.

3. AMAYA E., GIL F., FREIRE C., OLMEDO P., FERNÁNDEZ-RODRÍGUEZ M., FERNÁNDEZ M.F., OLEA N. Placental concentration of heavy metals in a mother-child cohort. Environ. Res. 120, 63, 2013.

4. STARSKA K., WOJCIECHOWSKA-MAZUREK M., MANIA M., BRULIŃSKA-OSTROWSKA E., BIERNAT U., KARŁOWSKI K. Noxious elements in milk and milk products in Poland. Pol. J. Environ. Stud. 20, (4), 1043, 2011.

5. TSUJI J.S., BENSON R., SCHOOF R.A., HOOK G.C. Health effect levels for risk assessment of childhood exposure to arsenic. Regul. Toxicol. Pharm. 39, 99, 2004.

6. IKEM A., NWANKWOALA A., ODUEYUNGBO S., NYAVOR K., EGIEBOR N. Levels of 26 elements in infant formula from USA, UK, and Nigeria by microwave digestion and ICP-OES. Food Chem. 77, 439, 2002.

7. LJUNG K., PALM B., GRANDÈR M., VAHTER M. High concentrations of essential and toxic elements in infant formula and infant foods - A matter of concern. Food Chem. 127, 943, 2011.

8. ZAND N., CHOWDHRY B.Z., WRAY D.S., POLLEN F.S., SNOWDEN M.J. Elemental content of commercial 'ready to-feed' poultry and fish based infant foods in the UK. Food Chem. 135, 2796, 2012

9. SARACOGLU S., SAYGI K.O., ULUOZLU O.D., TUZEN M., SOYLAK M. Determination of trace element contents of baby foods from Turkey. Food Chem. 105, 280, 2007.

10. MELO R., GELLEIN K., EVJE L., SYVERSEN T. Minerals and trace elements in commercial infant food. Food Chem. Toxicol. 46, 3339, 2008.

11. ZAND N., CHOWDHRY B.Z., ZATOR F.B., WRAY D.S., AMUNA P., PULLEN F.S. Essential and trace elements content of commercial infant foods in the UK. Food Chem. 128, 123, 2011.

12. Scientific Opinion of the Panel on Contaminants in the Food Chain (CONTAM) on a request from the European Commission on lead in food. The EFSA Journal 8, (4), 1570, 2010.

13. Joint FAO/WHO Expert Committee on Food Additives (JECFA). Evaluation of certain food additives and contaminants in food: $73^{\text {th }}$ Report of the Joint FAO/WHO Expert Committee on Food Additives. WHO Technical Report Series; No. 960, 2011

14. Scientific Opinion of the Panel on Contaminants in the Food Chain (CONTAM) on a request from the European Commission on cadmium in food. The EFSA Journal 980, 1139, 2009
15. Scientific Opinion of the Panel on Contaminants in the Food Chain (CONTAM) on arsenic in food. The EFSA Journal 7, 1351, 2009.

16. Joint FAO/WHO Expert Committee on Food Additives. Evaluation of certain contaminants in food: $72^{\text {th }}$ Report of the Joint FAO/WHO Expert Committee on Food Additives. WHO Technical Report Series; No. 959, 2011.

17. Scientific Opinion of the Panel on Contaminants in the Food Chain (CONTAM) on the risk for public health related to the presence of mercury and methylmercury in food. The EFSA Journal 10, 2985, 2012.

18. Methodology Publications of National Institute of Hygiene. "Method of determination of lead, cadmium, copper and zinc in foodstuffs by using FAAS technique." Warsaw, 1996 [In Polish].

19. Scientific Report of EFSA. Management of left-censored data in dietary exposure assessment of chemical substances. The EFSA Journal 8, 1557, 2010.

20. WOJCIECHOWSKA-MAZUREK M., STARSKA K., BRULIŃSKA-OSTROWSKA E., PLEWA M., BIERNAT U., KARŁOWSKI K. Monitoring of contamination of foodstuffs with elements noxious to human heath. Part I. Wheat cereal products, vegetable products, confectionery and products for infants and children (2004 year). Annals of the National Institute of Hygiene. 59, (3) 251, 2008 [In Polish with English abstract].

21. STARSKA K., WOJCIECHOWSKA-MAZUREK, MANIA M., REBENIAK M., KARŁOWSKI K. Contamination of food with lead and arsenic, In: Karłowski K., Rybińska K., Postupolski J. (Eds.), Exposure assessment to chemical and microbiological food contaminants - programme executed in the years 2004-2008. Warsaw, pp 4468, 2010 [In Polish].

22. Scientific Report of EFSA. Lead dietary exposure in the European population. The EFSA Journal 10, (7), 2831, 2012.

23. KAZI T.G., JALBANI N., BAIG J.A., AFRIDI H.I., KANDHRO G.A., ARAIN M.B., JAMALI M.K., SHAH A.Q. Determination of toxic elements in infant formulae by using electrothermal atomic absorption spectrometer. Food Chem. Toxicol. 47, 1425, 2009.

24. Food Surveys Information Sheets (FSIS). Survey of metals in weaning foods and formulae for infants. Food Survey Information Sheet No. 17/06, 2006.

25. Scientific Report of EFSA. Cadmium dietary exposure in the European population. The EFSA Journal 10, (1), 2551, 2012.

26. WOJCIECHOWSKA-MAZUREK M., STARSKA K., MANIA M., REBENIAK M., KARŁOWSKI K. Contamination of food with cadmium and mercury, in: Karłowski, K., Rybińska, K., Postupolski, J. (Eds.), Exposure assessment to chemical and microbiological food contaminants - programme executed in the years 20042008. Warsaw, pp. 13-43, 2010 [In Polish].

27. KAZI T.G., JALBANI N., BAIG J.A., ARAIN M.B., AFRIDI H.I., JAMALI M.K., SHAH A.Q., MEMON A.N. Evaluation of toxic elements in baby foods commercially available in Pakistan. Food Chem. 119, 1313, 2010.

28. HERNÁNDEZ-MARTİNEZ R., NAVARRO-BLASCO I. Estimation of dietary intake and content of lead and cadmium in infant cereals marketed in Spain. Food Control. 26, 6, 2012.

29. CARBONELL-BARRACHINA A.A., WU X., RAMÍREZGANDOLFO A., NORTON G.J., BURLÓ F., DEACON C., MEHARG A.A. Inorganic arsenic contents in rice-based infant foods from Spain, UK, China and USA. Environ. Pollut. 163, 77, 2012. 
30. HERNÁNDEZ-MARTÌNEZ R., NAVARRO-BLASCO I. Survey of total mercury and arsenic content in infant cereals marketed in Spain and estimated dietary intake. Food Control. 30, 423, 2013.

31. DA SACCO L., BALDASSARRE A., MASOTTI A. Diet's role in the toxicity of inorganic arsenic (iAs): A journey from soil to children's mouth. J. Geochem. Explor. 131, 45, 2013.

32. MEHARG A.A., SUN G., WILLIAMS P.N., ADOMAKO E., DEACON C., ZHU Y.G., FELDMANN J., RAAB A. Inorganic arsenic levels in baby rice are of concern. Environ. Pollut. 152, 746, 2008.

33. KARJALAINEN A.K., HALLIKAINEN A., HIROVONEN T., KIVIRANTA H., KNIP M., KRONBERGKIPPILÄ C., LEINO O., SIMELL O., SINKKO H., TUOMISTO J.T., VEIJOLA R., VENÄLÄINEN E.R., VIRTANEN S.M. Estimated intake levels for Finnish children of methylmercury from fish. Food Chem. Toxicol. 54, 70, 2013.
34. MANIA M., WOJCIECHOWSKA-MAZUREK M., STARSKA K., REBENIAK M., POSTUPOLSKI J. Fish and seafood as a source of human exposure to methylmercury. Annals of the National Institute of Hygiene. 63, (3) 257, 2012 [In Polish with English abstract].

35. MARTINS C., VASCO E., PAIXÃO E., ALVITO P. Total mercury in infant food, occurrence and exposure assessment in Portugal. Food Add. Contam. Part B. 6, (3), 151, 2013.

36. KSIĄŻYK J.B., WEKER H. New feeding plan for infants in Poland, since 2007 Contemporary Pediatrics, Gastroenterology, Hepatology and Child Feeding. 9, (4), 292, 2007.

37. PODSTAWA A., WITCZAK S. Metals and related substances in drinking waters in Poland. Warsaw, 2011 [In Polish].

38. MORISSET T., RAMIREZ-MARTINEZ A., WESOLEK N., ROUDOT A.C. Probabilistic mercury multimedia exposure assessment in small children and risk assessment. Environ. Int. 59, 431, 2013. 